\title{
CAN CONTRAST-ENHANCED MR IMAGING PREDICT SURVIVAL IN BREAST CANCER?
}

\author{
B. Boné ${ }^{1,2}$, B. K. Szabó ${ }^{2}$, L. G. Perbeck ${ }^{3}$, B. Veress ${ }^{4}$ and P. Aspelin ${ }^{1,2}$ \\ ${ }^{1}$ Department of Diagnostic Radiology, Huddinge University Hospital, ${ }^{2}$ Division of Diagnostic Radiology, Center for Surgical \\ Sciences, The Karolinska Institute, Huddinge University Hospital, ${ }^{3}$ Department of Surgery, Huddinge University Hospital, Huddinge \\ and ${ }^{4}$ Department of Pathology, University Hospital MAS, Malmö, Sweden.
}

\begin{abstract}
Purpose: To investigate the value of pre-operative contrast-enhanced MR imaging (CE-MRI) in predicting the disease-free and overall survival in breast cancer.

Material and Methods: The study population consisted of 50 consecutive patients with histopathologically verified primary breast cancer who preoperatively underwent CE-MRI examination between 1992 and 1993. A threetime point MR examination was performed where the enhancement rates $\left(C_{1}\right.$ and $\left.\mathrm{C}_{2}\right)$, signal enhancement ratio $\left(\mathrm{SER}=\mathrm{C}_{1} / \mathrm{C}_{2}\right)$ and washout $\left(\mathrm{W}=\mathrm{C}_{1}-\mathrm{C}_{2}\right)$ were calculated. The relation of these MR parameters to disease-free and overall survival was investigated. The median follow-up for surviving patients was 95 months. Univariate and multivariate statistical analyses were performed to evaluate the impact of different factors on prediction of survival.

Results: Of the MR parameters examined at univariate analysis, increased $\mathrm{C}_{1}(p=0.029), \mathrm{W}(p=0.0081)$ and SER values $(p=0.0081)$ were significantly associated with shorter disease-free survival, and only $\mathrm{C}_{1}(p=0.016)$ was related significantly to overall survival. Multivariate analysis for disease-free survival showed that the SER $(p=0.014)$ and tumor size $(p=0.001)$ were significant and independent predictors. Age $(p=0.003)$, lymph node status $(p=0.014)$, tumor size $(p=0.039)$ and proliferating cell nuclear antigen index $(p=0.053)$ remained independently associated with overall survival at multivariate analysis. $C_{1}$ was not confirmed as an independent predictor of overall survival.

Conclusion: Our findings support the presumption that CE-MRI is useful in predicting the disease-free survival in patients with breast cancer.
\end{abstract}

Key words: Breast cancer; MR; prognostic markers, survival analysis.

Correspondence: Botond K. Szabó, Department of Diagnostic Radiology, Huddinge University Hospital, SE-14186 Huddinge, Sweden. FAX +46858586249.

E-mail: botond.szabo@cfss.ki.se

Accepted for publication 20 February 2003.
The conventional TNM classification system for staging of breast cancer is widely used to determine treatment and provides prognostic information (1). The pre-operative staging is based on a combination of clinical and imaging findings and includes the assessment of tumor extent, multifocality and lymph node status. Mammography and breast ultrasonography, however, often underestimate the extent of the disease. MR imaging has been introduced as a complementary diagnostic tool for the detection of breast cancer. It is widely accepted that MR imaging can reveal mammographically occult breast malignancy, and has the ability to determine tumor size and exclude multifocality more accurately than standard breast imaging methods (2). Recently, MR imaging has also been suggested for pre-operative loco-regional staging, as well as for the evaluation of response to preoperative chemotherapy $(8,14)$.

Although staging is very important in the clinical management and contains information on tumor size and nodal involvement, treatment planning and prediction of prognosis cannot be made without the assessment of other postoperative morphologic variables such as histologic type and malignancy grade. Besides these classic prognostic factors, a number of biological markers have also 
been suggested to predict the clinical outcome of breast cancer (5).

In cancers other than breast cancer, it has been shown that dynamic contrast-enhanced MR imaging (CE-MRI) can contribute not only to establish the diagnosis, but also to identify patients with a poorer prognosis. Pharmacokinetic dynamic MR imaging was found to be a predictor of diseasefree survival in pediatric osteosarcoma (15). It has also been shown that MR imaging is able to assess tumor aggressiveness and offers information on the risk of local recurrence and on prediction of treatment success in cervical cancer $(9,12)$. Recent reports have demonstrated a correlation between MR findings and prognostic indicators in breast cancer $(3,13,16)$. However, there is no evidence to support the prognostic value of MR imaging concerning survival data in patients with breast cancer.

The purpose of our study was to investigate the value of pre-operative CE-MRI in predicting recurrence free and overall survival of breast cancer. We therefore evaluated the impact of different MR enhancement parameters on survival rates and compared it with established classic and molecular prognostic markers.

\section{Material and Methods}

Patients: In 1998, we published a study on 50 consecutively investigated breast cancer patients that had pre-operatively undergone CE-MRI between September 1992 and December 1993 (3). Inclusion criteria were a histopathologically verified primary breast malignancy, and detectable abnormality at MR imaging. The lesion had to be visible on MR on at least three consecutive images to avoid a partial volume effect when measuring the enhancement. This previous study was focused on the correlation of MR enhancement features and prognostic factors. We now decided to follow up on the disease-free interval and on survival in these patients and correlate this with different enhancement parameters derived from the MR measurements. The hospital medical records of the entire study population were reviewed, and diseasefree and overall survival was calculated. The median follow-up for surviving patients was 95 months (range, 23-111 months). The mean age of the patients at the diagnosis was 59 years (range, 29-83 years).

CE-MRI (1992-93): A three-time point examination was performed with one precontrast and two postcontrast series obtained $30 \mathrm{~s}$ to $7 \mathrm{~min}$ and 7 to 13 min, respectively, after the start of contrast agent injection, using a T1-weighted 3D fast low angle shot (FLASH) sequence on a $1.5 \mathrm{~T}$ system. Gd-DTPA was administered at a dose of $0.1 \mathrm{mmol} \mathrm{kg}^{-1}$ body weight. The enhancement rates $\left(C_{1}\right.$ and $\left.C_{2}\right)$ were calculated for the two postcontrast measurements. These parameters show the relative increase in signal intensity to the precontrast phase:

$$
C_{1}=\frac{S I_{1}-S I_{\text {pre }}}{S I_{\text {pre }}} \times 100 \quad C_{2}=\frac{S I_{2}-S I_{\text {pre }}}{S I_{\text {pre }}} \times 100
$$

where $C_{1}$ and $C_{2}$ are the early and late contrast enhancement ratios, $\mathrm{SI}_{\text {pre }}$ is the precontrast, and $\mathrm{SI}_{1}$ and $\mathrm{SI}_{2}$ are the first and second postcontrast signal intensities.

The washout (W) and signal enhancement ratio (SER) were also analyzed in this study. W was defined as the difference between the first and second enhancement rates:

$$
W=C_{1}-C_{2}
$$

The SER method calculates the ratio of early-tolate contrast enhancement, using the following equation:

$$
S E R=\frac{S I_{1}-S I_{\text {pre }}}{S I_{2}-S I_{\text {pre }}}=\frac{C_{1}}{C_{2}}
$$

This single parameter was used to describe the contrast agent kinetics for three-time point, highresolution MR imaging in a study conducted by ESSERMAN et al. (6).

The effect of the enhancement rates $\left(\mathrm{C}_{1}\right.$ and $\left.\mathrm{C}_{2}\right)$, $\mathrm{W}$ and SER variables on survival was investigated in this present study.

Histopathologic analysis: All surgically excised specimens were histopathologically examined. A series of pathologic features such as tumor size, axillary lymph node status, histologic type, mitotic count and nuclear grade were assessed. Data were also collected on the following biological markers: proliferating cell nuclear antigen (PCNA), factor VIII (FVIII), and CD 34.

The detailed morphologic analysis, immunohistochemical and microvessel counting methods have been described previously (3).

Statistical analysis: Disease-free survival was defined as the time interval between the primary operation and the local recurrence or metastasis or the last follow-up. Overall survival was the period from surgery until the date of death or the closure of the study. All observations of surviving patients and of those who died due to other causes than breast cancer were censored. Univariate survival analysis was based on the Kaplan-Meier product-limit method, and survival curves were compared using the log-rank test. The method described by HOTHORN \& LAUSEN (10) was used for the estimation of cut-points for continuous predictor variables. The maximally selected rank statistic for cut-points was calculated 
using the Maxstat software package for the $R$ statistical language and environment $(R$ 1.5.1 for Windows) (11).

Factors found to be significant at the univariate test were then selected for multivariate analysis. Cox proportional hazards regression was used for multivariate analysis to explore the relationship between prognostic factors. A $p$-value of less than 0.05 was considered as statistically significant. Univariate and multivariate statistical analyses were performed using the SPSS 9.0 for Windows (Statistical Package for Social Sciences, SPSS).

\section{Results}

Patient age and histopathologic characteristics of the lesions are summarized in Table 1.

The cumulative 5-year and 7-year survival rates for the whole cohort was $63 \%$ and $59 \%$ for disease-free, $81 \%$ and $77 \%$ for overall survival, respectively. Local recurrence or metastasis of the primary disease developed in $20(40 \%)$ patients. Until the last check-up, 15 (30\%) patients had died: $11(22 \%)$ women died from breast cancer, and $4(8 \%)$ patients from other causes.

Cut-point estimation: The cut-points of continuous variables that provide an optimum separation of patients into two groups with different survival times were estimated using the maximally selected rank statistics. The cut-off points of quantitative MR enhancement parameters for disease-free/overall survival were the following: $226 / 238$ for $C_{1}, 130.34 /$ 132.35 for $\mathrm{C}_{2}, 1.79 / 11.65$ for $\mathrm{W}$ and $1.008 / 1.028$ for SER. Figure 1 shows the standardized log-rank statistic as a function of the selected cut-point of SER for disease-free survival.

Analysis of disease-free survival: Table 2 shows the result of the univariate analysis. Among the different variables, $\mathrm{C}_{1}, \mathrm{~W}$, SER, tumor size, nuclear grade and the PCNA index were the statistically significant predictors of recurrence or metastasis. $\mathrm{C}_{2}$, patient age, nodal status, microvessel and mitotic counts had no significant impact on disease-free interval. Figures 2 and 3 show the Kaplan-Meier estimates of disease-free survival according to the MR enhancement parameters $\mathrm{C}_{1}$ and SER.

For disease-free survival, $\mathrm{C}_{1}, \mathrm{~W}, \mathrm{SER}$, tumor size, nuclear grade and the PCNA index were entered into a Cox proportional hazards regression model. Only SER and tumor size were found to be significant and independent prognostic factors of disease-free survival. $\mathrm{C}_{1}, \mathrm{~W}$, the PCNA index and nuclear grade were rejected from the final regression model (Table 3).

Analysis of overall survival: Assessing the effects of different parameters on overall survival, $\mathrm{C}_{1}$, age, tumor size, nodal involvement, nuclear grade and
Table 1

Patient age and histopathologic characteristics in 50 patients undergoing CE-MRI from 1992 to 1993

\begin{tabular}{|c|c|c|}
\hline Parameters & $\mathrm{n}=50$ & $\%$ \\
\hline \multicolumn{3}{|l|}{ Age } \\
\hline$\leq 50$ & 12 & 24 \\
\hline$>50$ & 38 & 76 \\
\hline \multicolumn{3}{|l|}{ Tumor size } \\
\hline$\leq 2 \mathrm{~cm}$ & 26 & 52 \\
\hline$>2 \mathrm{~cm}$ & 24 & 48 \\
\hline \multicolumn{3}{|l|}{ Histologic type } \\
\hline Ductal & 32 & 64 \\
\hline Lobular & 8 & 16 \\
\hline Other & 10 & 20 \\
\hline \multicolumn{3}{|l|}{ Nuclear grade } \\
\hline High & 23 & 46 \\
\hline Low & 27 & 54 \\
\hline \multicolumn{3}{|c|}{ Lymph node status } \\
\hline Positive & 17 & 34 \\
\hline Negative & 30 & 60 \\
\hline Data missing & 3 & 6 \\
\hline \multicolumn{3}{|l|}{ PCNA* } \\
\hline$\leq 43$ & 37 & 74 \\
\hline$>43$ & 13 & 26 \\
\hline \multicolumn{3}{|l|}{ Factor VIII* } \\
\hline$\leq 81$ & 38 & 76 \\
\hline$>81$ & 10 & 20 \\
\hline Data missing & 2 & 4 \\
\hline \multicolumn{3}{|l|}{ CD-34* } \\
\hline$\leq 55$ & 11 & 22 \\
\hline$>55$ & 33 & 66 \\
\hline Data missing & 6 & 12 \\
\hline \multicolumn{3}{|l|}{ Mitotic count* } \\
\hline$\leq 1$ & 17 & 34 \\
\hline$>1$ & 33 & 66 \\
\hline
\end{tabular}

*Cut-off points were calculated for overall survival by using the method of HOTHORN and LAUSEN (10). PCNA: proliferating cell nuclear antigen immunoreactivity; Factor VIII: microvessel count using factor VIII immunostaining; CD-34: microvessel count using CD-34 immunostaining.

the PCNA index were found to be significant at univariate analysis. $\mathrm{C}_{2}, \mathrm{~W}, \mathrm{SER}$, microvessel and mitotic counts failed to predict the overall survival in our patient cohort (Table 2).

Kaplan-Meier curves for overall survival, according to the MR enhancement parameters $C_{1}$ and SER are shown on Figs 4 and 5.

$\mathrm{C}_{1}$, age, tumor size, lymph node status, nuclear grade and the PCNA index were analyzed for overall survival in the second regression model. Age, lymph node status, tumor size and the PCNA index retained independent prognostic significance. $\mathrm{C}_{1}$ and nuclear grade were not significant (Table 4).

\section{Discussion}

To our knowledge, this paper is the first report that explores the prognostic significance of MR contrast enhancement in breast cancer using statistical methods of survival analysis. The result of our 


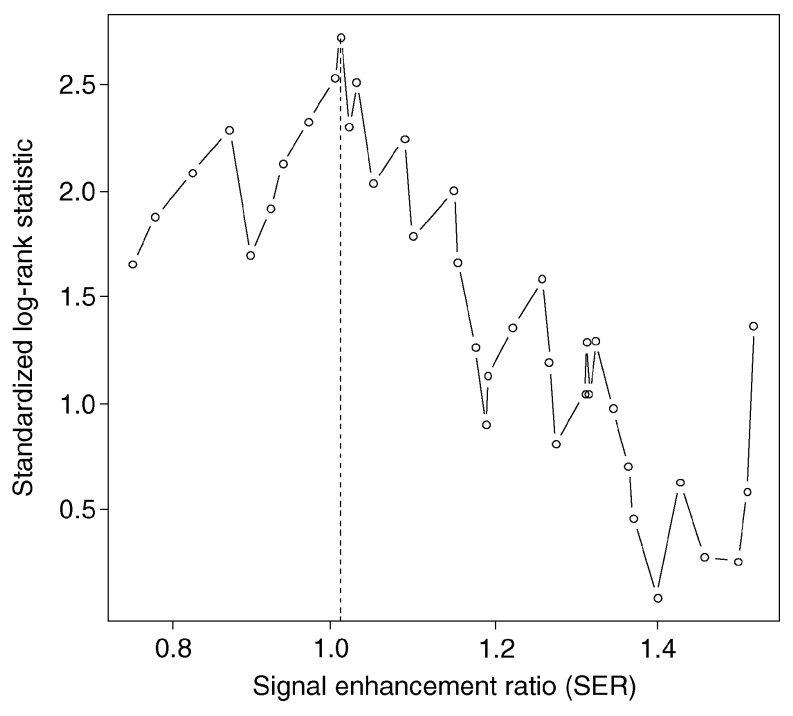

Fig. 1. Standardized log-rank statistics as a function of the chosen cut-point for the SER. The estimated cut-point is 1.008 for SER examining the disease-free survival time. Note - standardized logrank statistics based on the upper bound of the $p$-value according to Hothorn \& LAUSEN (10).

study demonstrates that SER measured by CEMRI using the three-time point temporal resolution is a significant and independent prognostic factor for the disease-free period in breast cancer patients. Although tumor size was found to be the strongest predictor for disease-free survival, the prognostic value of SER was superior to established prognostic factors such as nuclear grade and the PCNA index. On the univariate analysis, a significant difference in diseasefree and overall survival was observed for the first enhancement variable $\left(\mathrm{C}_{1}\right)$. Washout $\left(\mathrm{C}_{1}-\mathrm{C}_{2}\right)$ was also significant for disease-free survival. These MR parameters, however, were rejected from the Cox

\section{Table 2}

Univariate analysis on MR parameters and known prognostic factors in 50 patients with breast cancer

\begin{tabular}{lcc}
\hline Parameters & $\begin{array}{c}{ }^{1} p \text {-value for } \\
\text { disease-free survival }\end{array}$ & $\begin{array}{c}{ }^{1} p \text {-value for } \\
\text { overall survival }\end{array}$ \\
\hline $\mathrm{C}_{1}$ & $0.029^{*}$ & $0.016^{*}$ \\
$\mathrm{C}_{2}$ & 0.54 & 0.073 \\
$\mathrm{~W}$ & $0.0081^{*}$ & 0.067 \\
$\mathrm{SER}$ & $0.0081^{*}$ & 0.089 \\
$\mathrm{Age}$ & 0.36 & $0.011^{*}$ \\
Tumor size & $0.0073^{*}$ & $<0.0001^{*}$ \\
Nodal status & 0.064 & $0.0042^{*}$ \\
Nuclear grade & $0.024^{*}$ & $0.049^{*}$ \\
PCNA & $0.0009^{*}$ & $<0.0001^{*}$ \\
Factor VIII & 0.17 & 0.12 \\
CD-34 & 0.12 & 0.17 \\
Mitotic count & 0.12 & 0.067 \\
\hline
\end{tabular}

${ }^{1} p$-values estimated using the log-rank test. *Variables selected for multivariate analysis $(p<0.05) . C_{1}, C_{2}$ : first and second enhancement rates at CE-MRI; W: washout ( $\left.\mathrm{W}=\mathrm{C}_{1}-\mathrm{C}_{2}\right)$. Other abbreviations as in Table 1.

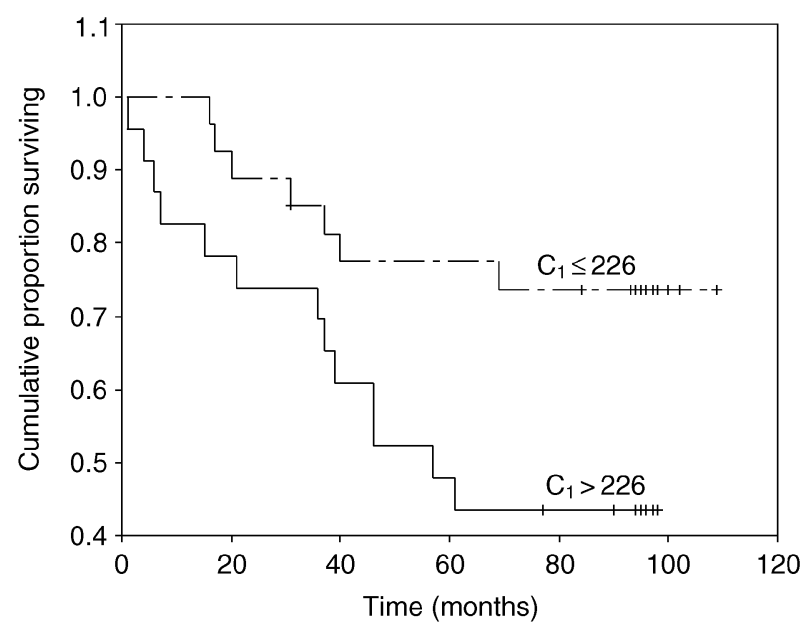

Fig. 2. Disease-free survival as a function of the early enhancement rate $\left(\mathrm{C}_{1}\right)$ at CE-MRI in 50 patients with breast cancer. The difference between the survival curves was significant (log-rank test, $p=0.029$ ).

regression models at multivariate analysis, and an explanation might be that the sample size of our patient population was relatively small to assess their real prognostic relevance. The second enhancement rate $\left(\mathrm{C}_{2}\right)$ failed to show any association with patients' survival times.

Signal intensities were measured at three-time points to obtain kinetic information on MR contrast agent uptake in this study. The enhancement rates $\left(\mathrm{C}_{1}\right.$ and $\left.\mathrm{C}_{2}\right)$ show the increase in signal intensity at postcontrast measurements compared with the precontrast values. Early enhancement $\left(\mathrm{C}_{1}\right)$ is often referred to as the "washin" phase, while $C_{2}$ is commonly used to obtain kinetic information. The degree of "washout" can be shown by the difference between $\mathrm{C}_{1}$ and $\mathrm{C}_{2}$.

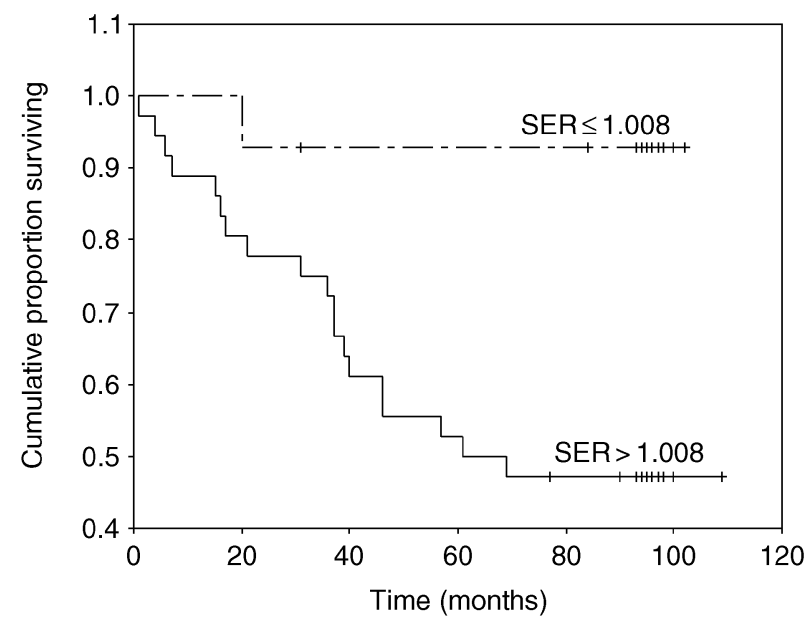

Fig. 3. Disease-free survival as a function of SER $\left(S E R=C_{1} / C_{2}\right)$ at CE-MRI in 50 patients with breast cancer. The difference between the survival curves was significant (log-rank test, $p=0.0081$ ). 
CE-MRI AND BREAST CANCER SURVIVAL

Table 3

Multivariate analysis* for disease-free survival in 50 patients with breast cancer

\begin{tabular}{lcccc}
\hline Parameters & $\begin{array}{c}\text { Hazard ratio } \\
\text { [exp }(\beta)]\end{array}$ & $\begin{array}{c}\text { Regression } \\
\text { coefficient }(\beta)\end{array}$ & $\begin{array}{c}\text { Standard error } \\
\text { for } \beta\end{array}$ & $\begin{array}{c}95 \% \text { confidence } \\
\text { interval for } \\
\exp (\beta)\end{array}$ \\
\hline SER & 11.79 & 2.47 & 1.00 & $1.66-83.84$ \\
Tumor size & 1.08 & 0.08 & 0.03 & $1.03-1.15$ \\
\hline
\end{tabular}

$\mathrm{C}_{1}, \mathrm{~W}, \mathrm{PCNA}$ and nuclear grade were rejected from the regression model. *Cox proportional hazards regression analysis was performed with the forward covariate selection method. Abbreviations as in Tables 1 and 2.

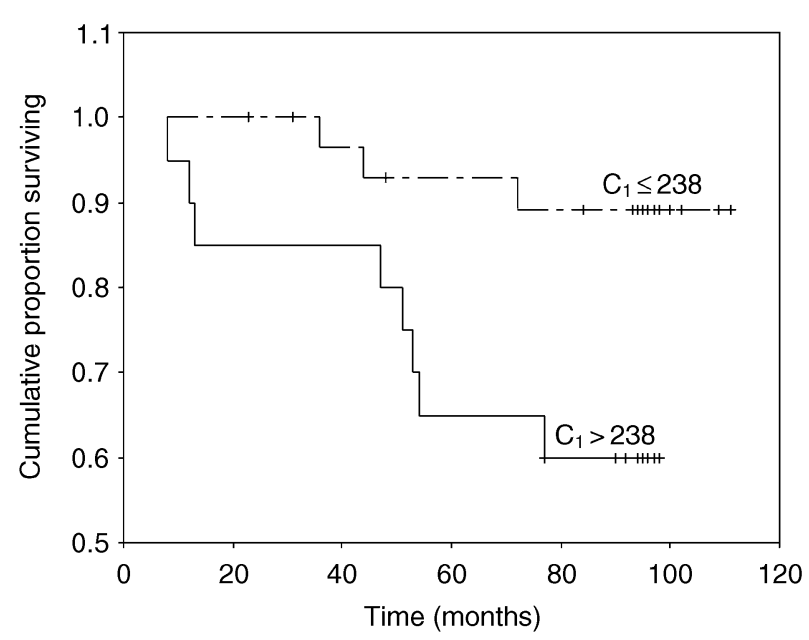

Fig. 4. Overall survival as a function of $\mathrm{C}_{1}$ at $\mathrm{CE}-\mathrm{MRI}$ in 50 patients with breast cancer. The difference between the survival curves was significant (log-rank test, $p=0.016$ ).

The SER analysis (6) has the ability to characterize both "washin" and "washout" in a single parameter. High SER values represent early enhancement and washout, while low SER values indicate slow enhancement and absence of washout. In this context, it is quite interesting that the threshold of $\mathrm{SER}=1$ (when $\mathrm{C}_{1}$ and $\mathrm{C}_{2}$ are equal) provides the best separation of patients with enhancing lesions into two prognostic groups (Fig. 1), and the group of lesions with SER values higher than 1 (quick enhancement and wash-

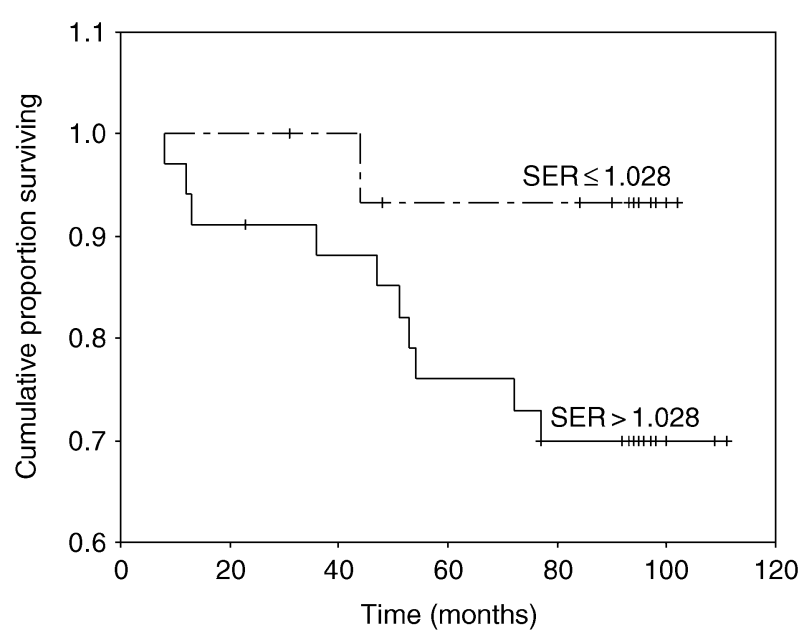

Fig. 5. Overall survival as a function of SER at CE-MRI in 50 patients with breast cancer. The difference between the survival curves was not significant (log-rank test, $p=0.089$ ).

out) represents the high-risk part of the population (Fig. 3).

Our previous study on the same patient material (3) reported a correlation between enhancement kinetics and established morphologic and molecular prognostic factors. Thus, MR enhancement was associated with the PCNA index and angiogenesis assessed by FVIII and CD-34. There was also a correlation between contrast enhancement and nuclear grade. Because recurrence free and overall

\section{Table 4}

Multivariate analysis* for overall survival in 50 patients with breast cancer

\begin{tabular}{lcccc}
\hline Parameters & $\begin{array}{c}\text { Hazard ratio } \\
\text { [exp } \beta]\end{array}$ & $\begin{array}{c}\text { Regression } \\
\text { coefficient, } \beta\end{array}$ & $\begin{array}{c}\text { Standard error } \\
\text { for } \beta\end{array}$ & $\begin{array}{c}\text { 95\% confidence } \\
\text { interval for } \\
\text { exp, } \beta\end{array}$ \\
\hline Age & 1.10 & 0.10 & 0.03 & $1.03-71.17$ \\
Lymph node status & 5.89 & 1.77 & 0.72 & $1.44-24.06$ \\
Tumor size & 1.07 & 0.07 & 0.03 & $1.00-1.14$ \\
PCNA & 1.09 & 0.09 & 0.05 & 0.003 \\
\hline
\end{tabular}

$\mathrm{C}_{1}$ and nuclear grade were rejected from the regression model. ${ }^{*}$ Cox proportional hazards regression analysis was performed with the forward covariate selection method. Abbreviations as in Tables 1 and 2. 
survival times were found to be significantly shorter in patients with strong MR contrast enhancement, this study seems to verify our preliminary assumption that there is an association between the extent of MR contrast uptake and breast cancer prognosis. In addition, our earlier findings are in agreement with other studies. Mussurakis et al. compared MR enhancement ratios with different histopathological prognostic factors (13), and found a strong association with lymph node status and histologic grade. STOMPER and colleagues reported an association between cell proliferating activity assessed by DNA S-phase percentage and peripheral MR enhancement pattern (16).

Our findings that tumor size, lymph node status and PCNA index are independent prognostic indicators are in accordance with previous larger studies $(4,17)$. In spite of the findings in our previous investigations, when the grade of angiogenesis was statistically related to the enhancement parameters, neither FVIII- nor CD-34-based vessel counts showed any association with disease-free and overall survival in the present study. This supports the view that morphologic evaluation of vascular density often fails to demonstrate an association with patients' survival due to the vascular heterogeneity of tumors (7).

There are recent studies focusing on the prognostic value of MR parameters in other types of malignant tumors. REDDICK et al. investigated the impact of a pharmacokinetic CE-MRI model on disease-free survival in pediatric osteosarcoma (15). The modeled exchange rate of the contrast agent between the vascular bed and extracellular space (e.g., vascular permeability) was highly predictive for the patients' disease-free period. According to their results, MR imaging has been proved to be an independent predictive factor in osteosarcoma after pre-operative chemotherapy. A study of MAYR et al. (12) showed that MR perfusion examinations might offer important information on treatment outcome in advanced cervical cancer. Hawighorst et al. (9) assessed the angiogenic activity of cervical cancer by conventional morphologic methods, and compared them with pharmacokinetic MR imaging. They found that functional MR parameters predicted the patients' survival, rather than did morphologic microvessel counts.

In conclusion, the result of this study suggests that CE-MRI can predict disease-free survival and is not only important for pre-operative diagnosis and staging, but may also be useful as a prognostic tool for the evaluation of patients with breast cancer. Despite our relatively small sample size, an association was also seen between MR enhancement and overall survival. On the basis of this promising result, further large-scale studies are needed to examine the impact of MR enhancement on the prediction of long-term overall survival.

\section{REFERENCES}

1. American Joint Committee on Cancer, American Cancer Society, American College of Surgeons: AJCC cancer staging manual, 5th edn. Lippincott-Raven 1997.

2. Boetes C, Mus RD, Holland R et al. Breast tumors: comparative accuracy of MR imaging relative to mammography and US for demonstrating extent. Radiology 1995; 197: 743-47.

3. Boné B, Aspelin P, Bronge L, Veress B. Contrast-enhanced MR imaging as a prognostic indicator of breast cancer. Acta Radiol. 1998; 39: 279-84.

4. Carter CL, Allen C, Henson DE. Relation of tumor size, lymph node status, and survival in 24,740 breast cancer cases. Cancer 1989; 63:181-87.

5. Elston CW, Ellis IO, Pinder SE. Review article. Pathological prognostic factors in breast cancer. Crit. Rev. Oncol. Hematol. 1999; 31: 209-23.

6. Esserman L, Hylton N, George T, Weidner N. Contrastenhanced magnetic resonance imaging to assess tumor histopathology and angiogenesis in breast carcinoma. Breast J. 1999; 5: 13-21.

7. Fridman V, Humblet C, Bonjean K, Boniver J. Assessment of tumor angiogenesis in invasive breast carcinomas: absence of correlation with prognosis and pathological factors. Virchows Arch. 2000; 437: 611-17.

8. Gilles R, Guinebretiere JM, Toussaint C et al. Locally advanced breast cancer: contrast-enhanced subtraction MR imaging of response to preoperative chemotherapy. Radiology 1994; 191: 633-38.

9. Hawighorst H, Weikel W, Knapstein PG et al. Angiogenic activity of cervical carcinoma: assessment by functional magnetic resonance imaging-based parameters and a histomorphological approach in correlation with disease outcome. Clin. Cancer Res.1998; 4: 2305-312.

10. Hothorn T, Lausen B. On the exact distribution of maximally selected rank statistics. Comp. Stat. Data Anal. xx 2003; xxx: (in press). Preprint available from: URL: http://www. mathpreprints.com/math/Preprint/hothorn/20020227/2.

11. Ihaka R, Gentleman R.R: A language for data analysis and graphics. J. Comp. Graph. Stat.1996; 5: 299-314.

12. Mayr NA, Yuh WT, Magnotta VA et al. Tumor perfusion studies using fast magnetic resonance imaging technique in advanced cervical cancer: a new noninvasive predictive assay. Int. J. Radiat. Oncol. Biol. Phys. 1996; 36: 623-33.

13. Mussurakis S, Buckley DL, Horsman A. Dynamic MR imaging of invasive breast cancer: correlation with tumour grade and other histological factors. Br. J. Radiol. 1997; 70: 446-51.

14. Orel SG, Schnall MD. MR imaging of the breast for the detection, diagnosis, and staging of breast cancer. Radiology 2001; 220: 13-30.

15. Reddick WE, Wang S, Xiong X et al. Dynamic magnetic resonance imaging of regional contrast access as an additional prognostic factor in pediatric osteosarcoma. Cancer 2001; 91: 2230-37.

16. Stomper PC, Herman S, Klippenstein DL, Winston JS, Budnick RM, Stewart CC. Invasive breast carcinoma: analysis of dynamic magnetic resonance imaging enhancement features and cell proliferative activity determined by DNA S-phase percentage. Cancer 1996; 77: 1844- 49.

17. Tahan SR, Neuberg DS, Dieffenbach A, Yacoub L. Prediction of early relapse and shortened survival in patients with breast cancer by proliferating cell nuclear antigen score. Cancer 1993; 71: 3552-59. 